\title{
MP-Polynomial Kernel for Training Support Vector Machines
}

\author{
Iván Mejía-Guevara ${ }^{1}$ and Ángel Kuri-Morales ${ }^{2}$ \\ ${ }^{1}$ Instituto de Investigaciones en Matemáticas Aplicadas y Sistemas (IIMAS), \\ Universidad Nacional Autónoma de México (UNAM), \\ Circuito Escolar S/N, CU, 04510 D. F., México \\ imejia@uxmcc2.iimas. unam.mx \\ ${ }^{2}$ Departamento de Computación, Instituto Tecnológico Autónomo de México, \\ Río Hondo No. 1, 01000 D. F., México \\ akuri@itam.mx
}

\begin{abstract}
In this article we present a new polynomial function that can be used as a kernel for Support Vector Machines (SVMs) in binary classification and regression problems. We prove that this function fulfills the mathematical properties of a kernel. We consider here a set of SVMs based on this kernel with which we perform a set of experiments. Their efficiency is measured against some of the most popular kernel functions reported in the past.
\end{abstract}

Keywords: MP-Polynomial Kernel, Kernel Methods, Support Vector Machine.

\section{Introduction}

Kernel methods have been a matter of study in the last years given their remarkable power and robustness to tackle nonlinear systems. Any kernel method solution comprises two parts: a) A module that performs the mapping into a high dimensional feature space and b) A learning algorithm designed to discover linear patterns in that space. Part (b) has been the focus of research in statistics and machine learning for decades, while part (a) rests on kernel functions. These kernel functions make it possible to represent linear patterns and ensure adequate representational power in higher-dimensional spaces [1. In particular, a SVM is a kernel method that has been successfully used in recent years for the solution of a multiplicity of practical applications.

The use of kernel functions in SVMs aims at the solution of non-linearly separable classification and non-linear regression problems. Some conditions must be fulfilled by those functions in order to be considered valid kernels. The main purposes of this article are: a) To propose a new polynomial kernel, b) To describe its implementation in SVMs and c) To compare its efficiency in the solution of classification and regressions tasks with some other kernels which have been used in previous applications of SVMs. In section 2, some important concepts regarding the characterization of kernel functions are discussed. In section 3 we

L. Rueda, D. Mery, and J. Kittler (Eds.): CIARP 2007, LNCS 4756, pp. 584-593. 2007.

(C) Springer-Verlag Berlin Heidelberg 2007 
review the main elements of an SVM for classification and regression problems. In section 4 a new kind of polynomial kernel is advanced and we prove that this kernel fulfills the conditions of section 2. Finally, in section 5, some experiments are performed to exemplify our approach. We close with some conclusions and future work considerations.

\section{Kernel Functions}

All the concepts and formal statements presented here were taken from [1, where a complete analysis of kernel methods and their applications is presented.

Definition 1 (finitely positive semi-definite functions). A function:

$$
\kappa: X x X \rightarrow R
$$

satisfies the finitely positive-definite property if it is a symmetric function for which the matrices formed by restriction to any finite subset of the space $X$ are positive semi-definite.

A formal characterization of a Kernel function is shown here as follows:

Theorem 1. (Characterization of kernels) A function

$$
\kappa: X x X \rightarrow R
$$

which is either continuous or has a countable domain, can be decomposed

$$
\kappa(x, z)=\langle\phi(x), \phi(z)\rangle
$$

into a future map $\phi$ into a Hilbert space $F$ applied to both its arguments followed by the evaluation of the inner product in $F$ if it satisfies the finitely positivedefinite property.

Some properties of Kernels, which are called closure properties [1, are as follows:

$$
\begin{aligned}
& \kappa(x, z)=\kappa_{1}(x, z)+\kappa_{2}(x, z) \\
& \kappa(x, z)=a \kappa_{1}(x, z) \\
& \kappa(x, z)=\kappa_{1}(x, z) \kappa_{2}(x, z) \\
& \kappa(x, z)=f(x) f(z) \\
& \kappa(x, z)=\kappa_{3}(\phi(x), \phi(z)) \\
& \kappa(x, z)=x^{T} B z
\end{aligned}
$$

where $\kappa_{1}$ and $\kappa_{2}$ are Kernels over $X \mathrm{x} X, X \subseteq R^{n}, a \in R^{+}, f($.$) a real valued$ function on $X, \phi: X \rightarrow R^{N}$ with $\kappa_{3}$ a kernel over $R^{N} x R^{N}$, and $\mathbf{B}$ a symmetric positive semi-definite $n_{x} n$ matrix.

The characterization of kernel functions, kernel matrices and the previous properties are needed to decide whether a candidate kernel is a valid one and to combine simple kernels to obtain more complex and useful ones. 


\section{Support Vector Machine}

In the last decade of the past century the SVM approach was discovered by [2] and has been studied and applied in multiple practical applications [3] [4] [5. A broad and conscientious exposition of this methodology can be found in [6] and [7].

\subsection{Classification}

In the case of binary pattern classification with SVM, a training sample $\tau=$ $\left\{x_{i}, d_{i}\right\}_{i=1}^{N}$ is considered where $x_{i}$ is the input pattern and $d_{i}$ is the target output. When attempting pattern classification, the goal is to find a hyper-surface that allows the separation of the objects in the sample into two classes: the first class should be on one side of the surface $d_{i}=1$ and the second class on the other side $d_{i}=-1$. The distance of the nearest points of both classes is called the margin of separation and the optimal surface considered to have been found when such margin is maximized. The optimal surface is known as Optimal Hyperplane (OHP) 2.

The objective of the SVM is to find the OHP for a given training set. This results in a constrained optimization problem. In practice a Quadratic Optimization Problem (QOP) is formulated in order to solve it. However, its dual formulation is more adequate, since only the Lagrange Multipliers (LMs) of the QOP need to be found. The dual form is as follows:

$$
\begin{aligned}
\operatorname{Max}_{\alpha} & \sum_{i=1}^{N} \alpha_{i}-\frac{1}{2} \sum_{i=1}^{N} \sum_{j=1}^{N} \alpha_{i} \alpha_{j} d_{i} d_{j} K\left(x_{i}, x_{j}\right) \\
\text { s.t. : } & \sum_{j=1}^{N} \alpha_{i}^{*} d_{i}=0 \\
& 0 \leq \alpha_{i} \leq C
\end{aligned}
$$

The solution of (5) is given by $\alpha_{i}, i=1,2, \ldots, N ; \mathrm{C}$ is known as the regularization parameter and appears in (5) as an upper bound for each $\alpha_{i}$. This parameter controls the trade-off between the complexity of the SVM and the number of acceptable misclassifications (a low value for $\mathrm{C}$ corresponds with a higher proportion of errors allowed in the solution, while fewer errors are permissible for high $\mathrm{C}$ values). The kernel function, $K\left(x, x_{i}\right)$, is used to construct a decision surface that is nonlinear in the input space but whose image in the feature space is linear. Once the solution of (5) is found, a function classifier is determined through the following expression:

$$
f(\mathbf{x})=\sum_{i=1}^{N} \alpha_{i} K\left(x, x_{i}\right)+b
$$

where the points corresponding with $\alpha_{i} \neq 0$ are called support vectors. 


\subsection{Nonlinear Regression}

In the case of nonlinear regression problems, the training set is analogous to the one used for classification problems; however, the values for $d_{i}$ are continuous. Given this training set, the goal of SVM is to find a function $f(\mathbf{x})$ such that $\left|f(x)-\hat{d}_{i}\right|<\epsilon$ for each element and a small $\epsilon$. In other words, errors are disregarded as long as they are smaller than a properly selected $\epsilon$. The function $f(\mathbf{x})$ has the a similar form as in the classification problems and it can also be found by solving a QOP. The dual form of this optimization problem is, as before, more appropriate. The dual formulation is as follows:

$$
\begin{aligned}
\operatorname{Max}_{\alpha, \alpha^{*}} & -\frac{1}{2} \sum_{i=1}^{N} \sum_{j=1}^{N}\left(\alpha_{i}-\alpha_{i}^{*}\right)\left(\alpha_{j}-\alpha_{j}^{*}\right) K\left(x_{i}, x_{j}\right) \\
& -\epsilon \sum_{i=1}^{N}\left(\alpha_{i}+\alpha_{i}^{*}\right)+\sum_{i=1}^{N} y_{i}\left(\alpha_{i}-\alpha_{i}^{*}\right) \\
\text { s.t. : } & \sum_{j=1}^{N}\left(\alpha_{i}-\alpha_{i}^{*}\right)=0 \\
& \alpha_{i} \alpha_{i}^{*} \in[0, C]
\end{aligned}
$$

The regularization parameter $C>0$ determines the tradeoff between the flatness of $f(x)$ and the acceptable number of points with deviations larger than $\epsilon$. The value of $\epsilon$ is inversely proportional to the number of support vectors $\left(\left(\alpha_{i}-\alpha_{i}^{*}\right) \neq 0\right)$ [7]. An adequate determination of $C$ and $\epsilon$ is needed for a proper solution. Their determination is explained in section 4 .

Once the solution of (7) is obtained, the support vectors are used to construct the following regression function:

$$
f(\mathbf{x})=\sum_{i=1}^{N}\left(\alpha_{i}-\alpha_{i}^{*}\right) K\left(x, x_{i}\right)+b
$$

We wish to emphasize that in both formulations (classification and regression) the kernel is used to train the SVM and to construct the classifier and regression function, respectively.

\subsection{Polynomial Kernel}

Many functions can be used as kernels, but only if they fulfill Mercer's theorem 8] and the conditions of section 2. Some of the most popular kernels discussed in the literature are the radial basis functions, the perceptrons and the algebraic polynomials. In this paper we focus on the last one and we compare its accuracy versus the one obtained with more complex polynomials.

$$
K\left(\mathbf{x}, \mathbf{x}_{i}\right)=\left(1+\mathbf{x} \cdot \mathbf{x}_{i}\right)^{p}
$$


For instance, if $\mathrm{m}=2$ and $\mathrm{p}=2$, equation (9) is as follows:

$$
K\left(\mathbf{x}, \mathbf{x}_{i}\right)=1+x_{1}^{2} x_{i 1}^{2}+2 x_{1} x_{2} x_{i 1} x_{i 2}+x_{2}^{2} x_{i 2}^{2}+2 x_{1} x_{i 1}+2 x_{2} x_{i 2}
$$

Of particular interest here is the degree of this kind of kernel which corresponds to the parameter $p$ in (9). The selection of this parameter is very important not only for SVMs but also for other approaches where polynomial kenels are used. This parameter determines the complexity of the model and affects its accuracy. For that reason, different approaches have been proposed in the past for its optimal selection [9] [10].

\section{MP-Polynomial Kernel}

Consider the following function:

$$
F(\mathbf{x})=\sum_{j_{1}}^{\rho} \sum_{j_{2}}^{\rho} \ldots \sum_{j_{m}}^{\rho} a_{j_{1}, j_{2}, \ldots, j_{m}}\left(x_{1}^{j_{1}} x_{2}^{j_{2}} \ldots x_{m}^{j_{m}}\right)
$$

We algebraically show that function (11) may be used as a valid kernel. The first disadvantage in trying to use this expression is that it holds as an argument an m-dimensional vector whereas a kernel requires two arguments. This problem was solved considering the following expression:

$$
K\left(\mathbf{x}, \mathbf{x}_{i}\right)=\sum_{j_{1}}^{\rho} \sum_{j_{2}}^{\rho} \ldots \sum_{j_{m}}^{\rho}\left(x_{1}^{j_{1}} x_{2}^{j_{2}} \ldots x_{m}^{j_{m}}\right) \cdot\left(x_{j 1}^{j_{1}} x_{i 2}^{j_{2}} \ldots x_{j m}^{j_{m}}\right)
$$

where $\mathbf{x}$ is a vector representing an element of the training set, with $m$ independent variables and $x_{i}$, the $i$-th object taken from this set. Notice that the functional structure of (11) and (12) is rather similar except for the term $\left(x_{1}^{j_{1}} x_{2}^{j_{2}} \ldots x_{m}^{j_{m}}\right)$, which was gotten from the elements of $x_{i}$, but that it may be considered as a constant. The structure of (12) now does indeed have a kernel structure. It just remains to be proven that it complies with the mathematical requirements, as is now established:

Proposition 1. The expression in (12) is a function with domain in $R^{m} x R^{m}$ and satisfies the required conditions for being a valid kernel.

Proof (of proposition). The first step in this proof relies on the observation that the expression in (12) may be represented in the form:

$$
K\left(\mathbf{X}, \mathbf{X}_{i}\right)=\left[X_{j 1} \otimes X_{j 2} \otimes \cdots \otimes X_{j m}\right]^{T} \cdot\left[X_{1} \otimes X_{2} \otimes \cdots \otimes X_{m}\right]
$$

where,

$$
X_{i}=\left(\begin{array}{c}
x_{i}^{0} \\
x_{i}^{1} \\
x_{i}^{2} \\
\cdots \\
x_{i}^{(1+p)^{m}}
\end{array}\right), X_{j i}=\left(\begin{array}{c}
x_{j i}^{0} \\
x_{j i}^{1} \\
x_{j i}^{2} \\
\cdots \\
x_{j i}^{(1+p)^{m}}
\end{array}\right)
$$


and $\otimes$ is Kronecker's matrix product. Therefore, expounding and applying the properties of a kernel we get:

$$
\begin{aligned}
K\left(\mathbf{X}, \mathbf{X}_{i}\right)= & {\left[X_{j 1} \otimes X_{j 2} \otimes \cdots \otimes X_{j m}\right]^{T} \cdot\left[X_{1} \otimes X_{2} \otimes \cdots \otimes X_{m}\right] } \\
= & \Phi\left(X_{j}\right)^{T} \cdot \Phi(X) \\
= & {\left[\Phi_{1}\left(X_{j}\right), \cdots, \Phi_{(1+p)^{m}}\left(X_{j}\right)\right] \cdot\left[\Phi_{1}(X), \cdots, \Phi_{(1+p)^{m}}(X)\right]^{T} } \\
= & \sum_{k=1}^{(1+p)^{m}} \Phi_{k}\left(X_{j}\right) \cdot \Phi_{k}(X) \\
& =\sum_{k=1}^{(1+p)^{m}} i_{k}^{T}\left[x_{j 1} \otimes x_{j 2} \otimes \cdots \otimes x_{j m}\right]^{T} \cdot i_{k}^{T}\left[x_{1} \otimes x_{2} \otimes \cdots \otimes x_{m}\right]
\end{aligned}
$$

where $i_{k}^{T}$ is a vector having $0^{\prime} s$ in all his positions except for the $k^{t h}$ one, in which it takes the value of 1 .

$$
K\left(\mathbf{X}, \mathbf{X}_{i}\right)=\sum_{k=1}^{(1+p)^{m}}\left(x_{j 1}^{[k]} \cdot x_{j 2}^{[k]} \cdots x_{j m}^{[k]}\right)^{T} \cdot\left(x_{1}^{[k]} \cdot x_{2}^{[k]} \cdots x_{m}^{[k]}\right)
$$

In (15) we find a scalar product which may be grouped in couples as $x_{j 1}^{[k]} x_{1}^{[k]}$ and from properties (4) the scalar product represents a kernel; in fact, it is the simplest possible one. Hence, we may put:

$$
K\left(\mathbf{X}, \mathbf{X}_{i}\right)=\sum_{k=1}^{(1+p)^{m}} \kappa\left(x_{j 1}^{[k]}, x_{1}^{[k]}\right) \cdots \kappa\left(x_{j m}^{[k]}, x_{m}^{[k]}\right)
$$

Finally, according to properties (4), the kernel product is in itself a kernel, so we have that:

$$
K\left(\mathbf{X}, \mathbf{X}_{i}\right)=\sum_{k=1}^{(1+p)^{m}} \kappa^{[k]}\left(x_{j}, x\right)
$$

This last expression is simple a summation of kernels which, from properties (4), also results in a valid kernel, which completes the proof.

We had called this new kernel as MP-Polynomial Kernel (MPK). A simple example of this kernel is as follows: if we assume two independent variables $(\mathrm{m}=2)$ and a degree $\mathrm{p}=2$. Then $K\left(\mathbf{X}, \mathbf{X}_{j}\right)=\left[X_{j 1} \otimes X_{j 2}\right]^{T} \cdot\left[X_{1} \otimes X_{2}\right]$, where:

$$
\begin{aligned}
X_{1} \otimes X_{2} & =\left[x_{1}^{0} x_{2}^{0}, x_{1}^{0} x_{2}^{1}, x_{1}^{0} x_{2}^{2}, x_{1}^{1} x_{2}^{0}, x_{1}^{1} x_{2}^{1}, x_{1}^{1} x_{2}^{2}, x_{1}^{2} x_{2}^{0}, x_{1}^{2} x_{2}^{1}, x_{1}^{2} x_{2}^{2}\right] \\
X_{j 1} \otimes X_{i 2} & =\left[x_{j 1}^{0} x_{i 2}^{0}, x_{j 1}^{0} x_{i 2}^{1}, x_{j 1}^{0} x_{i 2}^{2}, x_{j 1}^{1} x_{i 2}^{0}, x_{j 1}^{1} x_{i 2}^{1}, x_{j 1}^{1} x_{i 2}^{2}, x_{j 1}^{2} x_{i 2}^{0}, x_{j 1}^{2} x_{i 2}^{1}, x_{j 1}^{2} x_{i 2}^{2}\right]
\end{aligned}
$$

which yields:

$$
K\left(\mathbf{X}, \mathbf{X}_{i}\right)=\left(x_{1}^{0} x_{j 1}^{0}\right)\left(x_{2}^{0} x_{i 2}^{0}\right)+\ldots+\left(x_{1}^{2} x_{j 1}^{2}\right)\left(x_{2}^{2} x_{i 2}^{2}\right)
$$


Given this result, it turns out that it is possible to train an SVM using this new kernel. It only remains to verify the convenience of its adoption in practical cases. To this effect we performed a set of experiments regarding binary classification (i.e. the one involving only two classes) and non-linear regression. An important point in showing the validity of MPK is that its use is not limited to applications involving SVMs but, rather, it may also be applied as the basis for other kernel based methods.

\section{Experiments and Results}

It is well known that two key properties are required for a kernel to be used in practice 1. The first one requires that the kernel retains the measure of similarity appropriate to the particular task or domain. The second is that its evaluation should not be computationally intensive. The purpose of this section is determine experimentally if these properties are fulfilled by the MPK.

Two different tasks were tackled in this section: binary classification and regression. The dataset for classification was taken from UCI Machine Learning Repository. It refers to the identification of two kinds of diabetes. In case of regression, the data also has to do with diabetes, but now it refers to the study of the factors affecting patterns of insulin-dependent diabetes mellitus in children 1 . The diabetes classification (DC) dataset consist of 750 instances and 8 features. The diabetes-regression (DR) dataset has 43 cases and 2 features.

To analyze the performance of SVM in the solution of these tasks we used two popular kernels (polynomial in (9) and Gaussian) to compare their accuracy and efficiency with the MP-Polynomial Kernel.

In the case of classification, one statistical approach suggested by 11 was used. This test is known as $5 \mathrm{x} 2 \mathrm{cv}$ paired $t$ test, based in 5 iterations of 2 -fold cross-validation. In each replication of the test, the available data are partitioned into two equal-sized sets S1 and S2. The comparison of two different algorithms, for example RBF-SVM vs MPK-SVM2, is made by subtracting their corresponding error estimates in the application of 2-fold cross-validation in each replication. The means and variances $\left(s_{i}^{2}\right)$ are also successively estimated and finally a $t$ statistic is defined:

$$
\widetilde{t}=\frac{p_{(1)}^{1}}{\sqrt{\frac{1}{5} \sum_{i=1}^{5} s_{i}^{2}}}
$$

This is called $5 \times 2 \mathrm{vc} \tilde{t}$ statistic and it has an approximate $t$ distribution with 4 degrees of freedom. The value of $p_{(1)}^{1}$ represents the difference between the estimated errors for the two algorithms in the first replication. The comparison of two algorithms is based on the null hypothesis that there is no difference between their performances. The null hypothesis is rejected if $|\tilde{t}|>t_{4}$.

\footnotetext{
${ }^{1}$ This dataset can be downloaded from: http://www.liacc.up.pt/ ltorgo/Regression/DataSets.html

${ }^{2}$ RBF-SVM stands for an SVM trained with a Radial Basis Function kernel and similar to MPK-SVM.
} 
The results of the comparison of MPK-SVM vs the other kernels are shown in Table 1. It is important to remark that we used Matlab's program of [12] which was designed to train SVM for classification and regression problems. We modified this algorithm in order to compute the MPK.

The results of the tests for the diabetes classification problem are shown in Table 1 , where the values of the $\tilde{t}$ statistics for the hypothesis: 1) $\hat{p}_{p}-\hat{p}_{m p}=0$, 2) $\hat{p}_{p}-\hat{p}_{g}=0$ and 3) $\hat{p}_{m p}-\hat{p}_{g}=0$, where $\hat{p}_{p}, \hat{p}_{m p}$ and $\hat{p}_{g}$ are the estimated errors for PK-SVM 3 , MPK-SVM and RBF-SVM, respectively, are shown in the second column. The third column of Table 1 indicates that there is no statistical evidence to reject the null hypothesis in any case, i.e., there is no strong evidence supporting the idea that any algorithm is better than any other one. The parameter selection for the SVM was determined using 6-fold Cross Validation, so that the parameters for the polynomial kernels (MPK-SVM and PK-SVM) are $\mathrm{C}=16, \mathrm{p}=1$ and for the RBF-SVM are $\mathrm{C}=2, \sigma_{k}=0.6$. In the case of regression

Table 1. Hyphotesis tests for classification accuracy

\begin{tabular}{|l|c|c|}
\hline Test & t-statistic & Null Hyp \\
\hline \hline PK-SVM vs MPK-SVM & -0.12 & No reject \\
PK-SVM vs RBF-SVM & 0.41 & No reject \\
MPK-SVM vs RBF-SVM & 0.41 & No reject \\
\hline
\end{tabular}

problems, the models were chosen once the parameters $\epsilon, \mathrm{C}, \mathrm{p}$ and $\sigma$ were calibrated. Regarding the model trained with the radial basis kernel, the couple $[\mathrm{C}$, $\epsilon]$ was determined following the methodology proposed by [13, where the value of $\mathrm{C}$ is calculated as the maximum value resulting from $\left\{\bar{y}-3 * \sigma_{y}, \bar{y}+3 * \sigma_{y}\right\}$ and the computation of $\epsilon$ was determined by $\epsilon=\sigma \tau \sqrt{\ln (n) / n}$, being $\tau, \sigma$ and $\mathrm{n}$, a parameter set to 3 [13], the noise deviation and the number data train, respectively. The calculation of $\sigma$ required the computation of $\frac{\sum_{i=1}^{N}\left(y_{i}-\hat{y}_{i}\right)^{2}}{n-d}$; in this case, $\hat{y}$ represent the target values when a high order polynomial function is estimated using the training data. We applied the Genetic Multivariate approach 14 to estimate this polynomial and tried several degrees, choosing the one with the smallest Press statistic and the $\sigma$ according with the best parameter of the radial basis function gotten from the application of Leave-one-Out Cross Validation (LoOCV). The Press statistic is used for model selection and is obtained by adding the square of the residual errors that result from training the SVM $N$ times and leaving one observation out every time to determine the residual at every step 15. Actually, the Press statistic and the methodology of LoOCV are equivalent in this case, since for any particular observation the squared residual and the mean squared error for that observation are the same. The parameters obtained from this method were: $\mathrm{C}=6.9082, \sigma_{k}=0.6$ and $\epsilon=0.6779$

Since a similar analytical approach is not possible to calibrate $\mathrm{C}$ for polynomial kernels, we apply LoOCV for that purpose. We look for the best value that

${ }^{3}$ Stands for SVM trained with a classical polinomial kernel. 
belongs to this set $(1,2,4,8,16,32,64,128,254,512,1024)$. In the case of $\epsilon$ we used the value estimated for the radial basis function. The p parameter of the kernel function was chosen according with the lowest Press statistic (or LoOCV error) which reflects the best selection for the $\mathrm{C}$ parameter. The best values for $\mathrm{C}$ and $\mathrm{p}$ were 2 and 1 , respectively.

In table 2, the Press statistic indicates that the MP-polynomial performs as well as the classical polynomial kernel; RBF based SVM performs better than SVMs based on the other polynomials. $R^{2}$ (which is a measure of the expressive capabilities of the learning machine) also supports that result. The LoOCV error is presented in column 3; it yields similar results.

Table 2. Hyphotesis tests for regression accuracy

\begin{tabular}{|l|c|c|c|}
\hline Model & Press & $R^{2}$ & LoO error \\
\hline \hline PK-SVM & 14.4 & 0.34 & 0.34 \\
MPK-SVM & 14.5 & 0.33 & 0.34 \\
RBF-SVM & 12.5 & 0.43 & 0.29 \\
\hline
\end{tabular}

According to the results shown, it is possible to assert that MP-polynomial kernel performs as well as the alternative classical counterparts tested in this paper for both classification and regression problems. Moreover, this kernel can also be used to represent the decision or regression function, derived from the application of SVMs, as an algebraic explicit expression, in which the relationship among independent variables and their powers is straightforward. This result has been explored with succes in the past using traditional polynomial kernels, where the resulting algebraic structure was compared with the one gotten from the application of Multivariate Polynomial Analysis [14. However, two different structures were obtained. Fortunately, with the use of MPK is now possible to get the same algebraic stuctures with the use of those approaches and, hence, comparisons between them become also straighforward.

\section{Conclusions}

A new polynomial kernel (which we called "MP-Polynomial Kernel") is used to train a SVM in the solution of classification and regression problems. The characterization and mathematical proof of this function as a valid kernel was shown. The results of the experiments show that the use of this function for training SVMs is adequate in terms of accuracy. This conclusion is gotten by comparing its performance with other popular (polynomial and Gaussian) kernel functions. The statistical tests confirm this fact. An important advantage that we pointed out is the use of MP-polynomial kernel in the construction of an explicit algebraic expression as decision function of a SVM and its comparison with similar ones derived from other techniques. The use of this new kernel as a basis in applications with other kernel methods is a matter of future work. 


\section{References}

1. Shawe-Taylor, J., Cristianini, N.: Kernel Methods for Pattern Analysis. CUP (June 2004)

2. Boser, B.E., Guyon, I., Vapnik, V.: A training algorithm for optimal margin classifiers. In: COLT, pp. 144-152 (1992)

3. Blanz, V., Scholkopf, B., Bulthoff, H.H., Burges, C., Vapnik, V., Vetter, T.: Comparison of view-based object recognition algorithms using realistic $3 \mathrm{~d}$ models. In: ICANN, pp. 251-256 (1996)

4. Muller, K.-R., Smola, A.J., Ratsch, G., Scholkopf, B., K.J., Vapnik, V.: Predicting time series with support vector machines. In: ICANN, pp. 999-1004 (1997)

5. Weston, J., Gammerman, A., Stitson, M., Vapnik, V., Vovk, V., Watkins, C.: Density estimation using support vector machines (1997)

6. Burges, C.J.C.: A tutorial on support vector machines for pattern recognition. Data Mining and Knowledge Discovery 2(2), 121-167 (1998)

7. Smola, A., Schölkopf, B.: A tutorial on support vector regression (2004)

8. Haykin, S.: Neural networks: A comprehensive foundation. MacMillan, New York (1994)

9. Friedrichs, F., Igel, C.: Evolutionary tuning of multiple svm parameters. Neurocomputing 64, 107-117 (2005)

10. Min, J.H., Lee, Y.: Bankruptcy prediction using support vector machine with optimal choice of kernel function parameters. Expert Syst. Appl. 28(4), 603-614 (2005)

11. Dietterich, T.G.: Approximate statistical test for comparing supervised classification learning algorithms. Neural Computation 10(7), 1895-1923 (1998)

12. Gunn, S.: Support vector machines for classification and regression. Technical report (April 07, 1998)

13. Cherkassky, V., Ma, Y.: Practical selection of SVM parameters and noise estimation for SVM regression. Neural Networks 17(1), 113-126 (2004)

14. Kuri-Morales, A., Mejía-Guevara, I.: Analysis of algebraic expressions derived from genetic multivariate polynomials and support vector machines: A case study. In: Martínez-Trinidad, J.F., Carrasco Ochoa, J.A., Kittler, J. (eds.) CIARP 2006. LNCS, vol. 4225, pp. 974-984. Springer, Heidelberg (2006)

15. Rice, J.A.: Mathematical Statistics and Data Analysis. Duxbury Press, Good intermediary statistics book (1995) 